\title{
PVC Recognition for Wearable ECGs Using Modified Frequency Slice Wavelet Transform and Convolutional Neural Network
}

\author{
Zhongyao Zhao ${ }^{1,2}$, Xingyao Wang ${ }^{1}$, Zhipeng Cai ${ }^{1}$, Jianqing $\mathrm{Li}^{1}, \mathrm{Chengyu}^{\mathrm{Liu}}{ }^{1}$ \\ ${ }^{1}$ School of Instrument Science and Engineering, Southeast University, Nanjing, China \\ ${ }^{2}$ School of Electrical and Electronic Engineering, Nanyang Technological University, Singapore
}

\begin{abstract}
Progress in wearable techniques makes the long-term daily electrocardiogram (ECG) monitoring possible. Premature ventricular contraction $(P V C)$ is one of the most common cardiac arrhythmias. This study proposed a method by combining the modified frequency slice wavelet transform (MFSWT) and convolutional neural network (CNN). Training data are from the 2018 China physiological signal challenge (934 PVC and 906 non-PVC recordings). The first 10-s ECG waveforms in each recording were transformed into 2-D time-frequency images (frequency range of 0-50 Hz and size of $300 \times 100)$ using MFSWT. A 25-layer CNN structure was constructed, which includes five convolution layers with kernel size of $3 \times 3$, five dropout layers, five ReLU layers, five maximum pooling layers with kernel size of $2 \times 2$, a flatten layer, two fully connected layers, as well as the input and output layers. Test data were recorded from 12-lead Smart ECG vests, including $775 P V C$ and 742 non-PVC recordings. Results showed that, the proposed method achieved a high accuracy of $97.89 \%$ for PVC/non-PVC episodes classification, indicating that the combination of MFSWT and CNN provides new insight to accurately identify $P V C$ from the wearable ECG recordings.
\end{abstract}

\section{Introduction}

As a comprehensive reflection of cardiac activity, electrocardiogram (ECG) analysis has proven to be the archetypal method for detection of dangerous cardiac conditions. ECG effectively presents valuable clinical information regarding the rate, morphology, and regularity of the heart while being a low-cost and non-invasive test [1,2]. Advancement of wearable technology has enabled the recording of long-term dynamic ECGs. Specifically, wearable ECG analysis can be used for real-time detection of cardiac arrhythmias, such as premature ventricular contractions (PVCs).

PVCs result from irritated ectopic foci in the heart's ventricles, and are independent of the pace set by the sinoatrial node. Recent studies have shown that the occurrence of PVCs is indicative of increased risk of sudden cardiac death, and is linked to mortality when associated with myocardial infarction [3]. Consequently, their immediate detection and treatment is essential for patients with heart disease.

Different methods have been proposed for heart beat classification, such as support vector machine (SVM) [4,5], neural network [6,7], fuzzy mathematics [8], disease rule [9] and so on. Most PVC detection algorithms can obtain good classification performance on the standard ECG database [10]. However, the traditional neural network algorithm has a large amount of computation and a long training time, and it is hard to realize real-time detection. Fuzzy mathematics methods need to simplify many clinical discriminating rules and it is difficult to adapt to complex ECG records.

This study aims to explore the possibility of PVC identification method based on the combination of modified frequency slice wavelet transform (MFSWT) and convolutional neural network $(\mathrm{CNN})$. The idea is that with the highlight of PVC characteristic in 2-D MFSWT images, the employed CNN model can accurately identify the PVC incidents in ECGs.

\section{Methods}

\subsection{Data}

Training data were from the 2018 China physiological signal challenge (CSPC-2018) [11] and test data were recorded from a newly developed 12-lead Lenovo Smart ECG vest [12]. All recordings were intercepted the only first 10-s segments. The database consists of 1,840 training 10-s segments and 1,517 test segments. ECGs have a sampling rate of $400 \mathrm{~Hz}$ and a resolution of 16 bits. A large part of PVC signals only has a heart beat's abnormality, often lasting only about 1 second. Thus the 10-s ECG segments have been manually labeled by clinical experts and technicians as two types: PVC and non-PVC. The data profile was given in Table 1.

Table 1. Data profile in the training and test sets.

\begin{tabular}{lll}
\hline Database & Type & Number \\
\hline \multirow{2}{*}{ Training } & PVC & 934 \\
& non-PVC & 906 \\
\multirow{2}{*}{ Test } & PVC & 775 \\
& non-PVC & 742 \\
\hline
\end{tabular}




\subsection{Signal preprocessing}

Smooth denoising and normalization were used as signal preprocessing. Smooth denoising removes high frequency components from the signal and normalization can eliminate the dimensions of the sample and summarize the statistical distribution of the sample. This operation can improve the performance of learning speed rate of the neural network since the singular sample can not only increase the network training time, but also may cause the model fail to converge.

\subsection{Modified frequency slice wavelet transform (MFSWT)}

MFSWT can efficiently contain the time-frequency information of ECG in the transformed 2-D images, such as P-wave, QRS complex and T-wave, and were successfully applied in the previous studies [13,14]. A bound signal-adaptive frequency slice function (FSF) was introduced in MFSWT, which can realize the adaptive measurement of signal energy distribution at different observation frequencies. The reconstruction is readily accepted by clinicians. The model of MFSWT was expressed as follows:

$$
W_{f}(t, \omega)=\frac{1}{2 \pi} \int_{-\infty}^{+\infty} \hat{f}(k) \hat{p}^{*}\left(\frac{\mu-\omega}{q(f(k))}\right) e^{-i k t} d k
$$

where $\hat{f}(k)$ is the Fourier transform of $f(t), t$ and $\omega$ are observed time and frequency, respectively, $*$ represents conjugation operator, $\hat{p}$ is the FSF defined as $\hat{p}(x)=$ $e^{-x^{2} / 2}, q$ is a scale function of $\hat{f}(k)$. it uses the function form of Eq. (2):

$$
q=\delta+\operatorname{sign}(\nabla \mid \hat{f}(\mu))
$$

where $\delta$ is the frequency position of the signal main component. It can be estimated from the frequency position corresponding to maximum $|\hat{f}(\mu)| \cdot \nabla(\cdot)$ is a differential operator, and $\operatorname{sign}(\cdot)$ means signum function. FSF here adopts a Gaussian function form and $\hat{p}(0)=$ 1 is always true. Then the original signal can be reconstructed as follows:

$$
f(t)=\frac{1}{2 \pi} \int_{-\infty}^{+\infty} \int_{-\infty}^{+\infty} W_{f}(t, \omega) e^{i \omega(t-\tau)} d \tau d \omega
$$

Figure 1 shows the examples of preprocessed 10-s normal and PVC segments and their corresponding MFSWT images with different frequency ranges: $0-90 \mathrm{~Hz}$ and $0-50 \mathrm{~Hz}$ with a fixed pixel size of $300 \times 100$.

\subsection{Convolutional neural network (CNN)}

CNNs are now commonly used for deep learning tasks, voiding the need for any manual feature extraction and postprocessing. In the current study, a 25-layer $\mathrm{CNN}$ structure was constructed. Except the input and output layer, it includes five convolution layers, five dropout layers, five ReLU layers, five maximum pooling layers, a flatten layer and two fully connected layers. Figure 2 illustrates the architecture of the implemented network. Table 2 gives the specific parameter settings (after optimization) for the $\mathrm{CNN}$ architecture used in this study.
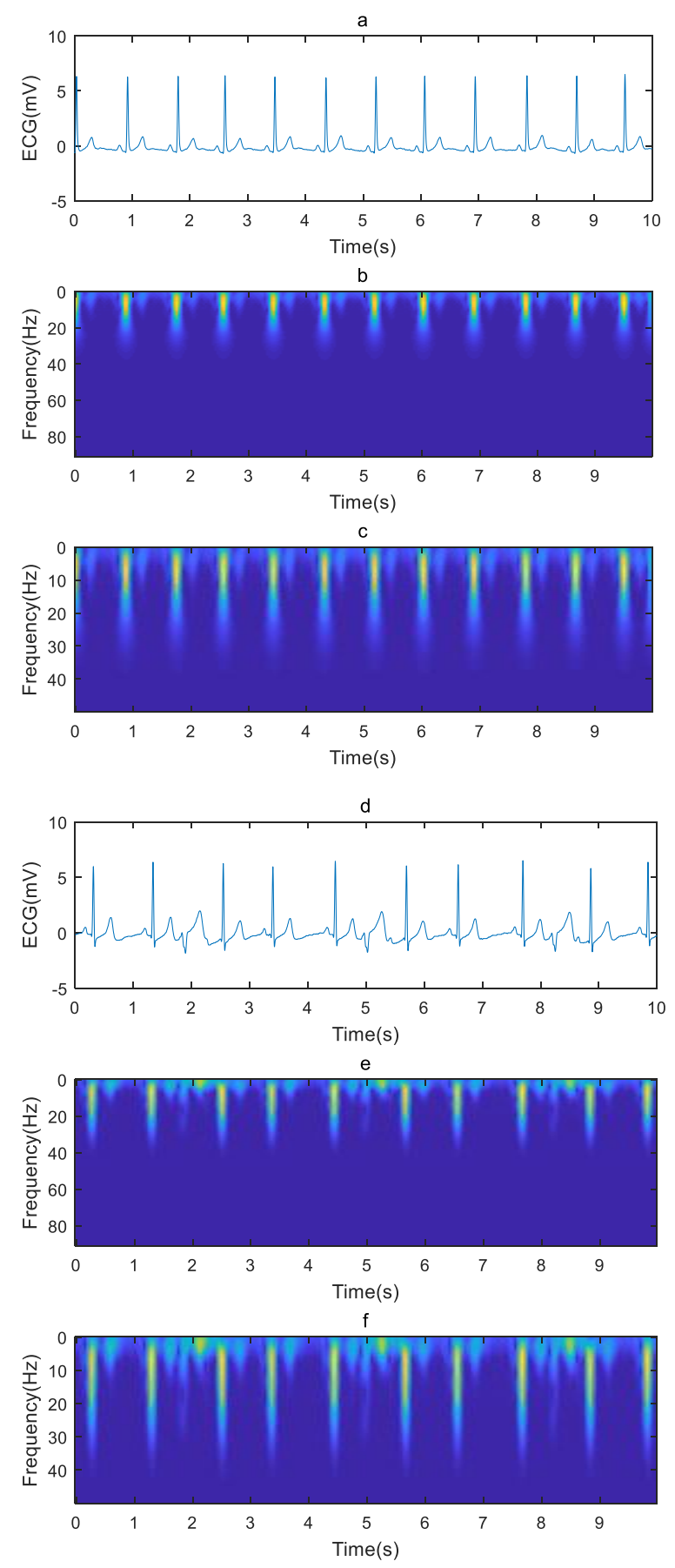

Figure 1. Examples from a 10-s normal ECG segment (a) and a 10-s PVC ECG segment (b), with their corresponding MFSWT spectra.

\subsection{Model evaluation}

Six widely used metrics, i.e., sensitivity $(\mathrm{Se})$, specificity $(S p)$, accuracy $(A c c)$, F-measure, area under the receiver operating characteristic curve (ROC), i.e., AUC, and Kappa coefficient, were used for evaluation. According to the labelled references, the result can generate four basic parameters: true positive $(T P)$, false positive $(F P)$, true negative $(T N)$ and false negative $(F N)$. In this case, $A c c=(T P+T N) /(T P+T N+F P+F N)$. $\mathrm{Se}$ is the true positive rate, is probability of incorrectly 

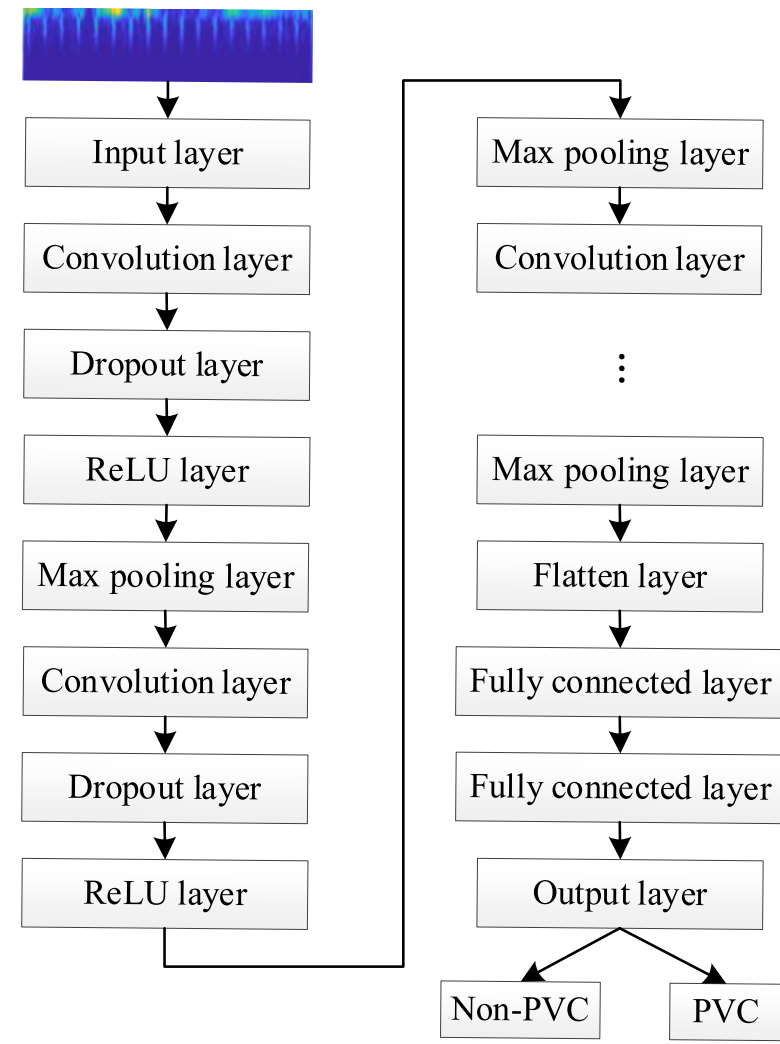

Figure 2. The architecture of the network.

diagnosing into positive among all positive patients, so $S e=T P /(T P+F N)$. $S p$ is proportion of incorrectly diagnosing into negative among all negative patients, so $S p=T N /(T N+F P)$.

F-measure is defined as:

$$
F_{\beta}=\frac{\left(\beta^{2}+1\right) P R}{\beta^{2} P+R}
$$

where $\beta$ is a parameter, $P$ is precision rate and it is defined as $P=\frac{T P}{T P+F P}, R$ is recall rate, and its value equals to $S e$. Take $\beta=1$, and we get $F_{1}=\frac{2 P R}{P+R}$.

Kappa coefficient can verify consistency and can be used to measure classification accuracy. It is defined as:

$$
K=\frac{p_{o}-p_{e}}{1-p_{e}}
$$

Table 2. CNN specifications designed for the ECG classification problem.

\begin{tabular}{ll}
\hline Parameters & Values \\
\hline Learning rate & 0.005 \\
Convolutional layer kernel size & $3 \times 3$ \\
No. of feature maps in the first convolutional & 16 \\
No. of feature maps in the second convolutional & 32 \\
No. of feature maps in the third convolutional & 64 \\
No. of feature maps in the fourth convolutional & 128 \\
No. of feature maps in the fifth convolutional & 256 \\
Leave probability of dropout layer & 0.5 \\
Max pooling layer kernel size & $2 \times 2$ \\
No. of neurons in the first fully connected layer & 256 \\
No. of neurons in the second fully connected layer & 2 \\
No. of epoch & 50 \\
Size of mini-batch & 10
\end{tabular}

where $p_{0}$ is Acc. Suppose that the number of real samples in each class is $a_{1}, a_{2}$ respectively, and the number of samples predicted in each class is $b_{1}, b_{2}$. The total number of samples is $n$, and $p_{e}=\frac{a_{1} \times b_{1}+a_{2} \times b_{2}}{n \times n}$.

ROC curve has the true positive rate $(\mathrm{Se})$ as the ordinate and the false positive rate $(1-S p)$ as the abscissa. AUC is defined as the area under the ROC curve.

\section{Results}

We equally split the 1,517 test ECG episodes into ten groups. The recording numbers for the ten groups are $151,151,151,152,152,152,152,152,152,152$ subsequently. Classification results from the evaluation metrics are summarized in Table 3 . We can see that all metrics reported relatively high scores, indicating that the developed model can efficiently identify the PVC rhythm from the non-PVC signals. An overall accuracy of $97.89 \%$ and an overall AUC of $97.88 \%$ were achieved, with less than $1 \%$ inter-group variability.

Table 3. Results from the evaluation metrics

\begin{tabular}{ccccccccccc}
\hline \multirow{2}{*}{ Fold } & \multicolumn{8}{c}{ Test data } \\
\cline { 2 - 11 } & TP & TN & FP & FN & $S e(\%)$ & $S p(\%)$ & $K(\%)$ & $F_{1}(\%)$ & $A c c(\%)$ & $A U C(\%)$ \\
\hline 1 & 72 & 76 & 2 & 1 & 98.63 & 97.44 & 96.02 & 97.96 & 98.01 & 98.03 \\
2 & 77 & 72 & 1 & 1 & 98.72 & 98.63 & 97.36 & 98.72 & 98.68 & 98.67 \\
3 & 73 & 73 & 2 & 3 & 96.05 & 97.33 & 93.38 & 96.69 & 96.69 & 96.69 \\
4 & 81 & 67 & 3 & 1 & 98.78 & 95.71 & 94.70 & 97.59 & 97.37 & 97.25 \\
5 & 85 & 66 & 1 & 0 & 100.0 & 98.51 & 98.66 & 99.42 & 99.34 & 99.25 \\
6 & 74 & 73 & 3 & 2 & 97.37 & 96.05 & 93.42 & 96.73 & 96.71 & 96.71 \\
7 & 81 & 68 & 3 & 0 & 100.0 & 95.77 & 96.03 & 98.18 & 98.03 & 97.89 \\
8 & 72 & 77 & 3 & 0 & 100.0 & 96.25 & 96.06 & 97.96 & 98.03 & 98.13 \\
9 & 73 & 76 & 3 & 0 & 100.0 & 96.20 & 96.06 & 97.99 & 98.03 & 98.10 \\
10 & 76 & 73 & 0 & 3 & 96.20 & 100 & 96.06 & 98.06 & 98.03 & 98.10 \\
\hline \multirow{2}{*}{ Total } & 764 & \multirow{2}{*}{721} & 21 & 11 & $98.58 \pm$ & $97.17 \pm$ & $95.78 \pm$ & $97.95 \pm$ & $97.89 \pm$ & $97.88 \pm$ \\
& & & & & 1.47 & 1.38 & 1.54 & 0.78 & 0.77 & 0.76 \\
\hline
\end{tabular}




\section{Discussion}

This study proposes an innovative framework for PVC detection based on MFSWT time-frequency representation and $\mathrm{CNN}$ classifier, which can accurately identify PVC ECG segments from the wearable big data ECGs. We build a unique $\mathrm{CNN}$ architecture to train the classification model that uses the time-frequency image generated by MFSWT method as input. The test data recorded from 12-lead Lenovo Smart ECG vest is evaluated by the trained model and obtain an accuracy of $97.89 \%$, indicating that the method has clinical significance. Xu et al. [15] applied this MFSWT method in the detection of atrial fibrillation, and enhanced the AF detection accuracy up to $84.85 \%$.

Yang et al. combined clinical diagnostic criteria with image processing methods, extracted heart rhythm parameters and QRS complex morphological features, and then used SVM to perform heart beat classification. The MIT-BIH database was used for training and verification and the $\mathrm{PVC}$ recognition rate was $95.31 \%$ [16]. It is noted that performance of the classifier relied too much on the quality of feature extraction. Since the morphologies of PVC beats can vary enormously from person to person, the model may suffer from overfitting and non-universality. A CNN method can eliminate the feature design and extraction process required in this kind of approaches.

In addition, considering that PVC is a dynamic process, real-time monitoring is important. Our work also has obvious advantage in the running time of the algorithm. $\mathrm{Li}$ et al. proposed a method to automatically discriminate PVC beats from other beats and artifacts with the use of wavelet transform and CNN. Ten-fold cross validation results on MIT-BIH showed that this algorithm achieved a highest overall accuracy of $97.96 \%$ using Paul wavelet [17]. However, it has been confirmed that MFSWT can better capture the tiny changes in the frequency domain than CWT [13]. Moreover, the ECG signal data used in our study were all from wearable ECG monitoring equipment so as to ensure clinical applicability. The next step of research should focus on expanding the types of diseases detected based on ensuring accuracy.

\section{Acknowledgement}

The study was partly supported by the National Natural Science Foundation of China (81871444), The authors thank the support from the Southeast-Lenovo Wearable Intelligent Monitoring Lab.

\section{References}

[1] Addison P. Wavelet transforms and the ECG: A review. Physiological Measurement. 2005; 26: R155-R199.

[2] Baroid SS. Willem Einthoven and the birth of clinical electrocardiography a hundred years ago. Cardiac Electrophysiology Review. 2003; 7: 99-104.

[3] Atsushi I, Hwa M, Hassankhani A, Liu T, Narayan SM. Abnormal heart rate turbulence predicts the initiation of ventricular arrhythmias. Pacing Clinical Electrophysiology.
2005; 11: 1189-1197.

[4] Ali K. Combining SVM and PSO for PVC detection. International Journal of Advances in Engineering Sciences. 2013; 3: 1-5.

[5] Huang D, Liao ZQ, Li CB, Li D, Huang WD. ECG PVC classification algorithm based on fusion SVM and wavelet transform. International Journal of Signal Processing, Image Processing and Pattern Recognition. 2015. 8: 193-201.

[6] Inan OT, Giovangrandi L, Kovacs GTA. Robust neural-network-based classification of premature ventricular contractions using wavelet transform and timing interval features. IEEE Transactions on Biomedical Engineering. 2006; 53: 2507-2515.

[7] Ankita M, Meena A. Detection of cardiac arrhythmias using different neural networks: A review. International Journal of Advanced Research in Computer and Communication Engineering. 2014; 3: 6992-6995.

[8] Meng ZH, Zhang YH, Bai J. A fuzzy method for classification of arrhythmias. Acta Electronica Sinica. 2001. 29: 1246-1248.

[9] Lek-uthai A, Ittatirut S, Teeramongkonrasmee A. Algorithm development for real-time detection of premature ventricular contraction. TENCON 2014-2014 IEEE Region 10 Conference, Bangkok. 2014; 1-5.

[10] Geeta K, Kumar DN, Kiran D. A review: detection of premature ventricular contraction beat of ECG. International Journal of Advanced Research in Electrical, Electronics and Instrumentation Engineering. 2015; 4: 939-942.

[11] Liu FF, Liu CY, Zhao LN, Zhang XY, Wu XL, Xu XY, et al. An open access database for evaluating the algorithms of ECG rhythm and morphology abnormal detection. Journal of Medical Imaging and Health Informatics. 2018; 8: $1368-1373$

[12] Liu CY, Zhang XY, Zhao LN, Liu FF, Chen XW, Yao YJ, et al. Signal quality assessment and lightweight QRS detection for wearable ECG SmartVest system. IEEE Internet of Things Journal. 2019; 6: 1363-1374.

[13] Luo K, Li JQ, Wang ZG, Cuschieri A. Patient-specific deep architectural model for ECG classification. Journal of Healthcare Engineering. 2017; Volume 2017: 4108720.

[14] Zhao ZY, Liu CY, Li YW, Li YX, Wang JY, Lin BS, et al. Noise rejection for wearable ECGs using modified frequency slice wavelet transform and convolutional neural networks. IEEE Access. 2019; 7: 34060-34067.

[15] Xu XY, Wei SS, Ma CY, Luo K, Zhang L, Liu CY. Atrial fibrillation beat identification using the combination of frequency slice wavelet transform and convolution neural networks. Journal of Healthcare Engineering. 2018; Volume 2018: 2102918.

[16] Yang X, Zhang Y. Arrhythmia recognition method based on support vector machine. Computer Engineering and Design. 2007; 18: 4442-4444.

[17] Li QC, Liu CY, Li Q, Shashikumar SP, Nemati S, Shen ZC, et al. Ventricular ectopic beat detection using a wavelet transform and a convolutional neural network. Physiological Measurement. 2019; 40: 5002.

Address for correspondence.

Chengyu Liu, $\mathrm{PhD}$

School of Instrument Science and Engineering

Southeast University, China

E-mail: chengyu@seu.edu.cn 